\title{
INFOCOVID: a socialização de saberes e informações científicas sobre covid-19 na rede social
}

\author{
Gênesis Vivianne Soares Ferreira Cruz ${ }^{1}$ \\ geviferreira@gmail.com \\ Closeny Maria Soares Modesto²; \\ soarescloseny@gmail.com \\ Stefany Pelegrini da Silva ${ }^{3}$; \\ universitario.pelegrini@gmail.com \\ Yara Rocha Luz; \\ yaraarluz@gmail.com \\ Yasmin Aynohan Sacal ${ }^{3}$ \\ yasmin.aynohan@gmail.com
}

\section{Resumo}

O objetivo do projeto de extensão "INFOCOVID" foi informar a população e profissionais de saúde através de dados científicos, utilizando uma linguagem acessível em diversas estratégias de alcance. Trata-se de um relato de experiência de ações de extensão mediadas por recursos virtuais e equipamentos eletrônicos, mantendo as recomendações de distanciamento social. 0 projeto foi divulgado no Instagram efetuando "posts, stories e lives" com diferentes conteúdos relacionados ao COVID-19. Considera-se que o projeto tem promovido a responsabilidade social de acadêmicos com a comunidade através da educação em saúde, reinventando meios de alcance e comunicação, com o propósito de promover o bem-estar e a saúde.

\section{Palavras-chave}

Coronavírus; Rede Social; Informações Científicas.

\footnotetext{
${ }^{1}$ Doutora em Enfermagem, Docente da Faculdade de Enfermagem, Universidade Federal de Mato Grosso, e-mail: geviferreira@gmail.com

${ }^{2}$ Mestranda em Ciências da Educação, Docente da Faculdade de Enfermagem, Universidade Federal de Mato Grosso, e-mail:

soarescloseny@gmail.com

${ }^{3}$ Acadêmicas da Faculdade de Enfermagem, Universidade Federal de Mato Grosso.
} 


\section{Introdução}

O coronavírus é uma família de vírus que provoca importantes infecções respiratórias que, em geral, produzem sintomas leves e moderados. No entanto, o agente da pandemia atual é uma nova cepa do vírus (SARS-CoV-2), que foi descoberta na China no final do ano de 2019 e que tem causado sintomas graves com vítimas fatais (BRASIL, 2020). Oficialmente, em fevereiro de 2020, a Organização Mundial de Saúde (OMS) denominou o agravo de "COVID-19", uma abreviação para Coronavirus Disease em referência ao ano de 2019. Os sintomas do COVID-19 podem aparecer após 2-14 dias de exposição e os principais sintomas são: febre, tosse e dificuldade ao respirar (CENTERS FOR DISEASE CONTROL AND PREVETION, 2020; WORD HEALTH ORGANIZATION, 2020).

As informações mais atualizadas e o conhecimento sobre a transmissão, a prevenção e o tratamento do COVID-19 podem trazer impactos diretos no controle da doença nas casas, no trabalho, nas escolas e nas comunidades, multiplicando saberes que podem alcançar outras pessoas (familiares, vizinhanças, etc.) evitando, assim, a propagação do vírus e interferindo na evolução da doença.

A atuação acadêmica neste contexto de pandemia pode favorecer o engajamento dos discentes em atividades direcionadas à comunidade, especialmente da enfermagem, com o potencial para promover a saúde e o bem-estar. Acredita-se que é possível estimular a relação academia/comunidade nessa complexa realidade, produzindo informações, trocas de saberes e estimulando a adoção de medidas que possam combater o avanço da pandemia, bem como trazer incremento à qualificação profissional. Além disso, em todas as ações universitárias busca-se a promoção da formação técnico-científica, ao inserir os alunos nos cenários de prática e de pesquisa, contribuindo para a formação em saúde/enfermagem.

As redes sociais cada vez mais fazem parte de vida em sociedade, ocupando um expressivo tempo de nossos dias, se tornaram muito mais do que ambientes de encontros e conversas, são canais de informação, de comunicação, de mídia, de atendimento e de relacionamento (PINTO; ANTUNES; ALMEIDA, 2020).

Na cultura contemporânea, são cada vez mais evidentes mudanças na forma como os indivíduos buscam informações sobre saúde, no relacionamento entre pacientes/comunidade e profissionais de saúde e nas interações sociais a respeito dos problemas e experiências em saúde. Tais mudanças são evidenciadas pelas novas formas e possibilidades de sociabilização proporcionadas pelas novas tecnologias, fenômeno denominado Cibercultura (FERNANDES; CALADO; ARAÚJO, 2018), e o desenvolvimento das ações do projeto possibilitou a reflexão sobre 
a importância do fortalecimento dessas estratégias com potencial para o empoderamento da população sobre esse novo fenômeno que foi trazido à tona de um forma mais abrangente com a pandemia.

As tecnologias de comunicação em saúde são ferramentas educativas que podem promover a articulação entre o meio acadêmico e a comunidade, desenvolvendo potenciais de autonomia e o respeito à cidadania (PINTO; ROCHA, 2016; PINTO; ANTUNES; ALMEIDA, 2020). Através das redes sociais pode-se oferecer informação, utilizando o espaço virtual para troca de saberes e conhecimentos (GIARETTA; GIULIO, 2018). No contexto de pandemia de COVID-19, surgiu, então, a iniciativa de abordar o tema "comunicação e saúde" com a finalidade de fornecer informações confiáveis à população, bem como incentivar atividades acadêmicas por meio de plataformas virtuais, valorizando as redes sociais.

Apesar das redes sociais estarem presente no cotidiano da maioria das pessoas, foi um desafio buscar informações confiáveis, oficiais e evidencias científicas num terreno movediço de abruptas descobertas científicas, constantes atualizações, disseminação de notícias falsas (fake news), incertezas e muita fragilidade da população, que ainda vive num clima de insegurança com relação ao tratamento e às descobertas de vacinas. Cada vez mais se vêm notícias sobre os avanços da pandemia pelo mundo e os dados epidemiológicos sobre internações, complicações e óbitos das vítimas.

0 acesso ilimitado e irrestrito às redes de informações, portanto, possui grande potencial de impactos negativos por permitir a veiculação de fake news sem possibilidade de checagens e verificações, o que pode gerar uma série de erros, medos e ansiedade; ao mesmo tempo que verifica-se na prática assistencial: precarização do atendimento às vítimas e a falta de estrutura das instituições de saúde, bem como a necessidade de uma atualização emergencial dos conhecimentos dos profissionais que integram as equipes de enfrentamento do COVID-19.

Diante deste cenário, o objetivo geral do projeto de extensão universitária "INFOCOVID" foi informar a população e profissionais de saúde através informações e dados científicos, utilizando uma linguagem acessível, em diversas estratégias de alcance: sínteses de artigos, documentários, notícias de fontes com referências confiáveis e verificáveis, elaboração de cartilhas e manuais técnicos, além da interação com o público, fomentando a troca de saberes, experiências e aprendizados.

Dentre os objetivos específicos, foram: realizar busca de evidências científicas para fundamentar as ações propostas; elaborar e organizar materiais educativos, vídeos, manuais e cartilhas voltados ao público-alvo; divulgar os materiais produzidos nas redes sociais: aplicativos de troca de mensagens e páginas sociais; realizar transmissões ao vivo (lives) em 
redes sociais para debater as estratégias de prevenção e combate do COVID-19; e distribuir materiais gráficos produzidos em instituições públicas hospitalares para profissionais de saúde.

\section{Métodos}

Trata-se de um relato de experiência descritivo sobre as atividades de extensão desenvolvidas por voluntários do projeto intitulado "INFOCOVID: ações educativas no combate ao COVID19", vinculado ao curso de enfermagem de uma universidade pública federal, desenvolvido no ano de 2020.

A equipe de trabalho do projeto foi composta por 02 docentes (coordenadora e vicecoordenadora) e 10 voluntários acadêmicos de enfermagem de diversos semestre de graduação. O planejamento, organização e a execução das ações de extensão foram mediadas por recursos virtuais (aplicativos de trocas de mensagens, redes sociais, salas virtuais, rede de internet) e equipamentos eletrônicos (celular e computador), mantendo as recomendações de distanciamento social, necessárias neste contexto apesar dos impactos sociais

As ações desenvolvidas foram compostas pelas seguintes etapas: *revisão de literatura e busca de evidências científicas mais atuais relacionadas ao tema proposto; *produção de textos/materiais/manuais com base na literatura científica; *elaboração de cartilhas, vídeos e materiais ilustrativos voltados à prevenção, manejo e tratamento do COVID19 para população em geral e profissionais de saúde; *divulgação dos produtos nas redes sociais; *realização de entrevistas e debates com profissionais de saúde da linha de frente de combate ao COVID-19.

Os encontros virtuais da equipe eram semanais, periodicamente duas vezes na semana pela plataforma Google meet, para definição e acompanhamento das atividades a serem desenvolvidas com posterior elaboração de relatório pelo Google forms. Os voluntários desenvolveram os materiais por meio de programas de software de acesso gratuito disponíveis na internet e aplicativos de smartphone, para a idealização, pesquisa, diagramação e edição dos produtos, sendo posteriormente revisados pelas orientadoras (docentes).

Os materiais gráficos (manuais/cartilhas/folders) foram solicitados à gráfica universitária, conforme autorização das instâncias superiores, sendo entregues nos serviços públicos hospitalares parceiros, com a finalidade precípua de que os mesmos possam ser utilizados como ferramenta de educação permanente em saúde.

As revisões de literatura foram feitas em bases científicas onde estão indexadas revistas nacionais e internacionais, identificando artigos com temas mais relevantes e pertinentes ao tema e para a produção de materiais informativos a serem postados na rede social on-line 
Instagram. Para a criação da arte dos posts foi utilizado o Canva, uma ferramenta de desenvolvimento de designe gráfico com acesso gratuito por smartphone e computadores.

$\mathrm{Na}$ plataforma Instagram também foram realizadas transmissões ao vivo com enfermeiros que atuam na linha de frente do combate ao COVID-19, em formato de entrevista (lives), com o intuito de relatar suas experiências na assistência direta aos pacientes infectados, respondendo perguntas do público on-line sobre a temática.

O público-alvo atingido foi de profissionais e acadêmicos de saúde, população em geral, internautas, que possuíssem acesso às plataformas gratuitas das redes sociais.

\section{Resultados e Discussão}

O projeto INFOVOCID foi divulgado como página do aplicativo social Instagram, nomeado como “@infocovidᄀ_enfufmt” (Figura 1) que, até o momento, possui 301 seguidores e já efetuou 143 publicações (posts) com diferentes conteúdos relacionados ao COVID-19, entre eles: recomendações sobre a saúde mental e física, características clínicas da doença, alimentação saudável, estresse infantil durante a pandemia, saúde indígena e população negra, informativos e atualizações sobre a doença, séries: "mito ou verdade?" e "você sabia?", utilizando várias estratégias de postagens e "stories" através de imagens gráficas ilustrativas e interativas.

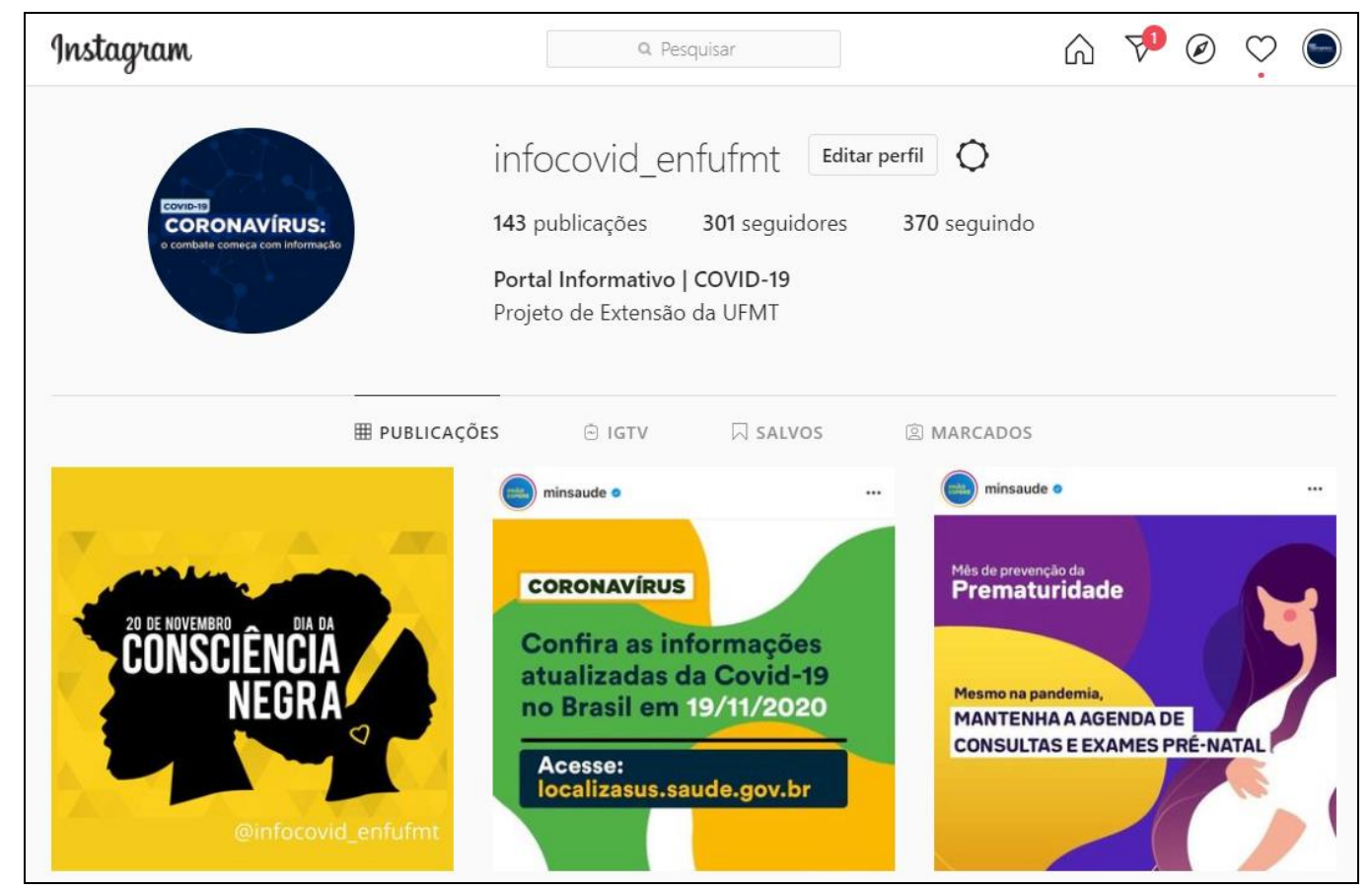

Figura 1: Página "Infocovid" no Instagram.

Fonte: Elaborado pelos autores. 
Outra forma de interagir na plataforma, foi através das lives (entrevistas ao vivo) mediadas por docentes que, geralmente, ocorreram no período noturno, com audiência em média de 40 pessoas simultaneamente on-line. Para essa ação foi exigida uma fase de preparação para os envolvidos na busca pelo desenvolvimento de habilidades de comunicação e de relações interpessoais, bem como o manejo dos recursos tecnológicos, demandados para a ambiência virtual.

No total, até o momento, ocorreram 09 lives que obtiveram entre 51 e 118 visualizações, com duração média de 1 hora. Os convidados entrevistados foram enfermeiros que atuavam na linha de frente do cuidado ao paciente com COVID-19 em diversas áreas de atuação (UTI adulto, neonatal e pediátrica; hospitais de referência; policlínicas e SAMU), utilizando um roteiro de entrevistas (Figura 2).

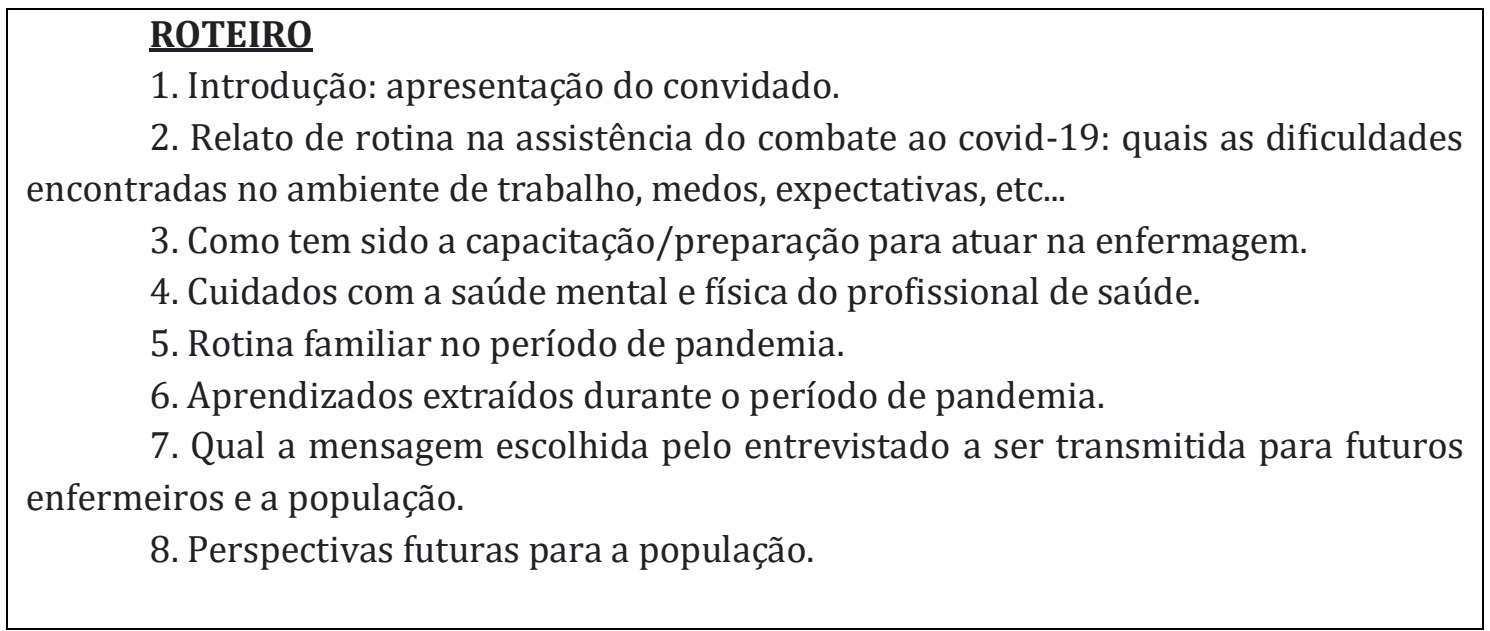

Figura 2: Roteiro de entrevista em "lives" realizadas na página do Instagram.

Fonte: Elaborado pelos autores.

Acredita-se que essa modalidade de comunicação/interação foi a de maior alcance nessa plataforma social, visto que a interação entre o público e os entrevistados foi maior do que nas publicações (posts). Durante as lives, os enfermeiros relatavam suas rotinas de assistência aos pacientes com COVID-19 e as perguntas feitas on-line abrangeram temas como: o uso de medicamentos, manejo clínico, cuidados na interação social, recomendações e protocolos da Organização Mundial da Saúde (OMS) e Ministério da Saúde. Assim, o chat on-line (um espaço para comentários dos internautas) possibilitou a troca de informações e a comunicação mais direta sobre a temática.

A ferramenta "stories" do Instagram também foi muito utilizada para divulgar e enfatizar as novas publicações, anúncios e divulgação das lives, além de promover stories interativos 
através de enquetes, perguntas e testes sobre a temática COVID-19, que serviram para subsidiar o alcance dos objetivos propostos assim também como direcionar a escolha de novas temáticas.

Foi produzido um "Manual de cuidados a pacientes críticos com COVID-19" e uma coletânea de materiais técnicos direcionado aos profissionais da saúde, cuja publicação será viabilizada através da editora universitária. A construção desses materiais foi idealizada e elaborada pelos alunos voluntários no projeto e alunos da graduação, remotamente, sendo revisado pelos docentes, incluindo a diagramação e ilustração de um encarte que depois de impresso será distribuído aos enfermeiros em assistência direta aos pacientes com COVID-19 em hospitais públicos parceiros do município.

No decorrer da execução remota das ações extensão em "tempos de COVID-19", foi possível perceber o "passar" por uma experiência com potencial para ampliar não só o universo de nossos saberes técnicos formais de uma área específica, como a enfermagem, mas também alavancar o desenvolvimento de habilidades e competências para a utilização e manejo de aplicativos e software ainda não utilizados no processo de ensino-aprendizagem, pois abruptamente, as ações de extensão diretas com a comunidade e aulas presenciais foram substituídas para meios remotos, obrigando professores e alunos a um aprendizado rápido de novas tecnologias de comunicação e informação (TICs) (AMEM; NUNES, 2006; GIARETTA; GIULIO, 2018).

Essa nova forma de ensinar, aprender e executar veio para preencher um espaço vazio que surgiu com o distanciamento social, que no seu início se caracterizou como um contexto ainda desconhecido para muitos dos atores sociais envolvidos, mas com o passar do tempo e o engajamento de grupos sociais (PINTO; ANTUNES; ALMEIDA, 2020; FERNANDES; CALADO; ARAÚJO, 2018), feito de forma bastante colaborativa.

Para a equipe executora das ações desta extensão universitária as dificuldades foram sendo superadas no "decorrer delas", no "aprender fazendo", dando lugar às experiencias cada vez mais desafiadoras, e após cada uma das atividades realizadas eram feitas reuniões de avaliação para que se discutisse os aspectos positivos e também os negativos que geravam novos desafios/aprendizados para a superação necessária.

Neste cenário, os recursos tecnológicos, particularmente as Tecnologias de Informação e Comunicação (TIC), tiveram papel central tanto para a ampliação de processos de participação social dos voluntários (GIARETTA; GIULIO, 2018), como para a possível inovação de ações educativas bem diferenciadas das comumente realizadas antes da pandemia, trazendo novas configurações sociais, educacionais, profissionais, comunicacionais e políticas. 
Também é importante considerar os impactos diretos provocados pelo contexto da pandemia do coronavírus na vida em sociedade, pois trouxe consigo duas realidades conflitantes: a convivência com o agente causador do agravo, que assolou o mundo inteiro nos últimos meses; e a necessidade de distanciamento e resiliência da população, em todos os sentidos, sendo necessária a prevenção da doença através da implementação de cuidados pessoais (higienização das mãos, do ambiente, de vestimentas e de utensílio) e adoção de uma nova rotina e novos hábitos de vida.

Acredita-se que a socialização de saberes e informações confiáveis e seguras favorece a promoção da saúde e o desenvolvimento da resiliência necessária para o enfrentamento do processo de adoecimento, além de uma maior e melhor adesão às medidas de prevenção, sendo considerada uma importante ferramenta do cuidado comunitário (PINTO; ROCHA, 2016).

De modo geral, considera-se que o projeto INFOCOVID teve obtido êxito em seus objetivos ao atingir a população e os profissionais da saúde por meio de plataformas virtuais com informações claras e confiáveis (científicas), assim também como contribui para minimizar os impactos da pandemia no cotidiano das pessoas e dos atores que integram a linha de frente de combate a COVID-19.

Além disso, em sua proposta tem promovido a responsabilidade social de acadêmicos com a comunidade através de métodos inovadores de educação em saúde, reinventando meios de alcance e comunicação, com o propósito de promover o bem-estar e a saúde, tornando possível que a academia (universidade) cumpra com o seu papel social - uma das bases necessárias para a operacionalização da integração do tripé ensino-serviço-comunidade.

No entanto, reconhece-se seus limites, especialmente quanto ao seu alcance, pois construir uma ampla rede de interações com alto nível de engajamento requer tempo e impulsionamento orgânico. Portando, ainda é preciso pensar em estratégias cada vez mais abrangentes e acessíveis, como o uso de outras plataformas sociais (como Facebook e Twitter), para obter maior alcance de internautas e divulgação dos produtos gerados pela extensão.

\section{Conclusão}

Através das redes sociais vimos o crescente interesse a população sobre a temática abordada, pois a pandemia trouxe sérios prejuízos não só para a saúde da população, como também para a economias dos países, com grandes impactos no cotidiano das famílias e na qualidade de vida das pessoas, causando sofrimentos psíquicos e emocionais. 
Dentre as mudanças sociais mais drásticas, encontram-se as novas formas de ensinar, aprender e socializar as informações que são importantes para a população em geral. A rede de notícias e informações se propagam velozmente ultrapassando as fronteiras das mídias tradicionais e alcançando cada vez mais os meios virtuais de relacionamento, incluindo aplicativos de trocas de mensagens instantâneas e páginas de interação social.

Com o distanciamento social, no meio acadêmico, os docentes e discentes tiveram que adquirir competências e habilidades no manejo de tecnologias virtuais para o ensino/aprendizagem remota, enfrentando um período de adaptação da ambiência virtual do ensino.

Com relação aos efeitos práticos dessas modalidades de ação, acredita-se que a rede de socialização de saberes e informações tem possibilitado, sobretudo, o potencial de minimizar os riscos decorrentes do processo pandêmico que vivemos neste cenário de incertezas, resultando em escolhas mais conscientes, adoção de hábitos saudáveis, contribuindo para a redução da curva epidemiológica (à longo prazo). Tais efeitos podem ser alcançados com engajamento de todos os atores envolvidos e com ampla participação social, com o compromisso e a responsabilidade necessários para a consolidação do papel social da academia (universidade).

Esperamos que essa semente que foi plantada no contexto atual, possa ser cultivada futuramente quando a pandemia passar, pois isso pode dar visibilidade a uma nova forma de fazer a enfermagem e alcançar os objetivos traçados quanto a utilização de estratégias de educação em saúde, bem como para a implementação dos cuidados inovadores em enfermagem nos diversos contextos de atuação, individualizados e comunitários.

\section{Referências}

BRASIL, Ministério da Saúde. 0 que é coronavírus? Brasília, 2020. Disponível em: https://coronavirus.saude.gov.br/

FERNANDES, Larissa de Siqueira; CALADO, Camila; ARAUJO, Claudia Affonso Silva. Redes sociais e práticas em saúde: influência de uma comunidade online de diabetes na adesão ao tratamento. Ciênc. saúde coletiva, Rio de Janeiro, v. 23, n. 10, p. 3357-3368, Oct. 2018.

ORGANIZAÇÃO PAN-AMERICANA DE SAÚDE (OPAS). Mensagens e ações importantes para a COVID-19 Prevenção e controle em escolas. Março de 2020. Disponível em: https://iris.paho.org/bitstream/handle/10665.2/51955/OPASBRACOVID1920015 por.pdf?seq uence=1\&isAllowed=y. Acesso em: 15 Ago. 2020.

GIARETTA, Juliana Barbosa Zuquer; GIULIO, Gabriela Marques Di. O papel das tecnologias de comunicação e informação (TIC) no urbano do século XXI e na emergência dos novos 
movimentos sociais: reflexões a partir de experiências na megacidade de São Paulo. Rev. Bras. Estud. Urbanos Reg., São Paulo, v. 20, n. 1, p. 161-179, Apr. 2018. Disponível em:

$<$ http://www.scielo.br/scielo.php?script=sci_arttext\&pid=S2317-

$15292018000100161 \& \operatorname{lng}=$ en\&nrm=iso $>$. Acesso em 04 Nov. 2020.

AMEM, Bernadete Malmegrim Vanzella; NUNES, Lena Cardoso. Tecnologias de Informação e Comunicação: contribuições para o processo interdisciplinar no ensino superior. Rev. bras. educ. med., Rio de Janeiro, v. 30, n. 3, p. 171-180, Dec. 2006. Disponível em:

$<$ http://www.scielo.br/scielo.php?script=sci_arttext\&pid=S0100-

55022006000300008\&lng=en\&nrm=iso >. Acesso em 23 Ago. 2020.

PINTO, Luiz Felipe; ROCHA, Cristianne Maria Famer. Inovações na Atenção Primária em Saúde: o uso de ferramentas de tecnologia de comunicação e informação para apoio à gestão local. Ciênc. saúde coletiva, Rio de Janeiro, v. 21, n. 5, p. 1433-1448, maio 2016. Disponível em <http://www.scielo.br/scielo.php?script=sci_arttext\&pid=S1413$81232016000501433 \& \operatorname{lng}=$ pt\&nrm=iso $>$. Acesso em 14 nov. 2020.

PINTO, Pâmela A.; ANTUNES, Maria João L.; ALMEIDA, Ana Margarida P. O Instagram enquanto ferramenta de comunicação em saúde pública: uma revisão sistemática. 15th Iberian

Conference on Information Systems and Technologies (CISTI), Seville, Spain, 24 - 27 June 2020. Disponível em:

<https://www.researchgate.net/profile/Pamela_Pinto/publication/342966660_Instagram_as_a _communication_tool_in_public_health_a_systematic_review/links/5f1058a545851512999e95a a/Instagram-as-a-communication-tool-in-public-health-a-systematic-review.pdf $>$. Acesso em 20 Nov. 2020. 
INFOCOVID: la socialización del conocimiento y la información científica sobre covid-19 en la red social

\section{Resumen}

El objetivo del proyecto de extensión "INFOCOVID" fue informar a la población ya los profesionales de la salud a través de datos científicos, utilizando un lenguaje accesible en varias estrategias de divulgación. Se trata de un relato de experiencia de acciones de extensión mediadas por recursos virtuales y equipos electrónicos, manteniendo las recomendaciones de distancia social. El proyecto se publicó en Instagram realizando "posts, stories y lives" con diferentes contenidos relacionados con COVID19. Se considera que el proyecto ha promovido la responsabilidad social de los académicos con la comunidad a través de la educación para la salud, reinventando los medios de alcance y comunicación, con el propósito de promover el bienestar y la salud.

\section{Palabras clave}

Coronavirus, Red Social, Información Científica.
INFOCOVID: the socialization of knowledge and scientific information about covid-19 in the social network

\section{Resumo}

The objective of the "INFOCOVID" extension project was to inform the population and health professionals through scientific data, using an accessible language in several outreach strategies. This is an experience report of extension actions mediated by virtual resources and electronic equipment, maintaining the recommendations of social distance. The project was published on Instagram making "posts, stories and lives" with different contents related to COVID-19. It is considered that the project has promoted the social responsibility of academics with the community through health education, reinventing means of reach and communication, with the purpose of promoting well-being and health.

\section{Key words}

Coronavirus, Social Network, Scientific Information. 